\title{
Antisymmetry and channel coupling contributions to the absorption for $\mathrm{p}+\alpha / \mathrm{d}+{ }^{3} \mathrm{He}$
}

\author{
S.G. Cooper \\ Physics Department, The Open University, \\ Milton Keynes MK7 6AA, UK
}

\begin{abstract}
To understand recently established empirical $\mathrm{p}+\alpha$ potentials, RGM calculations followed by inversion are made to study contributions of the $\mathrm{d}+{ }^{3} \mathrm{He}$ reaction channels and deuteron distortion effects to the $\mathrm{p}+\alpha$ potential. An equivalent study of the $\mathrm{d}+{ }^{3} \mathrm{He}$ potential is also presented. The contributions of exchange non-locality to the absorption are simulated by including an phenomenological imaginary potential in the RGM. These effects alone strongly influence the shape of the imaginary potentials for both $\mathrm{p}+$ $\alpha$ and $\mathrm{d}+{ }^{3} \mathrm{He}$. The potentials local-equivalent to the fully antisymmetrisedcoupled channels calculations have a significant parity-dependence in both real and imaginary components, which for $\mathrm{p}+\alpha$ is qualitatively similar to that found empirically. The effects on the potentials of the further inclusion of deuteron distortion are also presented. The inclusion of a spin-orbit term in the RGM, adds additional terms to the phase-equivalent potential, most notably the comparatively large imaginary spin-orbit term found empirically.
\end{abstract}

March 21, 2018 


\section{Introduction}

In successive studies, application of Iterative Perturbative (IP) inversion to empirical $\mathrm{p}+\alpha S$-matrices has established a local potential, which is both parity and energy dependent, covering a wide energy range from subthreshold energies up to $\sim 70 \mathrm{MeV}$, [1, 2]. For energies above the inelastic threshold, parity dependence is also necessary in the imaginary components to reproduce the empirical behaviour of $|S(l, j)|$. Furthermore, a recent energy dependent inversion for $\mathrm{p}+{ }^{16} \mathrm{O}$ found an imaginary parity dependent component essential to reproduce satisfactorily a comprehensive set of elastic cross-section and analysing power data, [3]. Parity dependence in the real $\mathrm{N}+\alpha$ potential has been widely studied and is understood to simulate particular exchange effects, notably heavy-particle pickup, arising through the antisymmetry, «. At subthreshold energies, a close correspondence has been established between the real potential local equivalent to Resonating Group Model (RGM) phase shifts and parity dependent potentials determined by inversion from empirical phase shifts, [5, 6]. It is now of interest to know how far fully antisymmetrised coupled channels calculations can explain the form of imaginary parity dependent components found empirically and this is a major aim of the present study. In including coupling to the $\mathrm{d}+{ }^{3} \mathrm{He}$ channel, the $\mathrm{d}+{ }^{3} \mathrm{He} S$-matrices are also calculated and a parallel study of the $\mathrm{d}+{ }^{3} \mathrm{He}$ potential is made possible.

This study is based on RGM calculations followed by IP $S(l) \rightarrow V(r)$ inversion. The 5 nucleon system has been subject to many microscopic investigations (for example, [7]- [13]). At subthreshold energies the single channel approximation provides qualitatively reasonable results, while at higher energies, increasing numbers of reaction channels are required. Inevitably, such calculations will always necessitate some unknowns or approximation, for example in the cluster wavefunction basis or the nucleon nucleon potential, so that precise fit to experimental results remain difficult to achieve. Even the most elaborate of recent RGM calculations, [12, with up to 25 cluster configurations, cannot reproduce the $\mathrm{p}+\alpha$ reaction cross-sections. No attempt is then made here to reproduce the empirical potentials precisely and the current aim is to establish general features in the potential which arise through the non-locality of the antisymmetry and channel coupling. The only coupling included is between the $\mathrm{p}+\alpha$ and $\mathrm{d}+{ }^{3} \mathrm{He}$ channels and with the excited deuteron pseudo-states, which represent s-wave deuteron breakup following Ref. [11. These RGM calculations do not include a tensor force, which is expected to be of greatest importance at energies close to the $\mathrm{d}+{ }^{3} \mathrm{He}$ threshold, [13], The current calculations are made at energies well above the $\mathrm{d}+{ }^{3} \mathrm{He}$ threshold.

At the simplest level, absorption effects have been simulated by introducing a phenomenological imaginary potential into the single channel RGM (see for example Ref. [8]). The absorption in the resulting $S(l)$ will be modified by the non-locality due to exchange contributions, and the imaginary potential resulting from subsequent $S(l) \rightarrow V(r)$ inversion may differ substantially from the phenomenological imaginary potential. As will be shown, this nonlocality can even lead to the imaginary potential becoming emissive at the 
origin. These induced effects depend on the system studied and, in Sect. 3, results are compared for $\mathrm{p}+\alpha$ and $\mathrm{d}+{ }^{3} \mathrm{He}$ scattering.

The outline of the paper is as follows: Section 2.1 outlines details of the RGM plus inversion procedure and establishes the notation used in the rest of the paper. The results of using an imaginary potential in single channel RGM calculations are presented in Section 3. The contributions of the deuteron distortion channels alone to the $\mathrm{d}+{ }^{3} \mathrm{He}$ potential are discussed in Section 4 and the results of including reaction channels into the RGM for both $\mathrm{p}+\alpha$ and $d+{ }^{3}$ He are described in Section 5 . The paper ends with a short summary section.

\section{RGM plus inversion calculations}

\subsection{The RGM calculations}

The RGM calculations use a modified form of the codes of Blüge et al, [14]. The original code permits RGM calculations in both single channel mode and with RGM channel coupling between the $\mathrm{p}+\alpha$ and $\mathrm{d}+{ }^{3} \mathrm{He}$ channels. Deuteron distortion effects may additionally be included using the pseudo-state method of Kanada et al, [15]. The code of Blüge et al has been rewritten in Fortran 90 and adapted to incorporate an alternative choice of nucleon-nucleon potential, an imaginary phenomenological potential and a larger basis for the deuteron wavefunction, which consequently permits the inclusion of more pseudo-states. The code cannot incorporate a tensor interaction in the nucleon-nucleon force, so that the reaction channel coupling is allowed only between $\mathrm{p}+\alpha$ and the $\mathrm{d}$ $+{ }^{3}$ He configuration with channel spin $s=1 / 2$, i.e. there can be no coupling to the $\mathrm{d}+{ }^{3} \mathrm{He}$ channel with $s=3 / 2$. However, calculations without reaction channel coupling are presented for the $\mathrm{d}+{ }^{3} \mathrm{He}$ spin $3 / 2$ channel, to allow a direct comparison of the potentials for the two channel spins.

In the RGM calculations presented here, the internal motions of the $\alpha$ and ${ }^{3} \mathrm{He}$ are described by single gaussian form-factors of widths, 0.606 and 0.367 respectively. These widths give reasonable estimates for the root mean square matter radii of the respective nuclei, but the corresponding energy expectation values are -24.72 and $-3.51 \mathrm{MeV}$ respectively for the $\alpha$ and ${ }^{3} \mathrm{He}$, a little greater than the experimental values. These ground state energies put the $\mathrm{d}+{ }^{3} \mathrm{He}$ threshold at $19.0 \mathrm{MeV}$, again slightly higher than the experimental value of $18.36 \mathrm{MeV}$, [16]. However, since a precise agreement with empirical results is not the aim of this work, this disagreement should not be very significant. The deuteron wavefunction is represented by an 8 gaussian function, [17, which was constructed to have several low energy deuteron excitation modes, [18]. The corresponding ground and excited deuteron energies are $-2.202,-0.036$, $0.2008,0.888$ and $3.94 \mathrm{MeV}$, and 4 orthogonal pseudo-states may be used in calculations to represent deuteron distortion.

All the RGM calculations are based on the nucleon-nucleon potential of Thompson et al, 19], with the exchange mixture parameter $u=0.95$. For simplicity, most of the results presented in the following sections are obtained 
without a spin-orbit interaction. Where specifically stated, calculations include the nucleon-nucleon spin-orbit force of Reichstein and Tang, [7].

The following notation is used to simplify the discussion in the rest of this paper: Single channel calculations, leading to real phase shifts and potentials are denoted "SC". RGM calculations which include reaction channel coupling only, without distortion effects, are denoted "CC". The $\mathrm{d}+{ }^{3} \mathrm{He}$ calculations with coupling to deuteron pseudo-states only are labelled "DC" and the complete calculations, in which both the $\mathrm{p}+\alpha$ and $\mathrm{d}+{ }^{3} \mathrm{He}$ channels are coupled to the four deuteron pseudo-states are denoted "6C".

An imaginary central potential can be included in the RGM calculations as a direct addition to the real RGM direct potential, following the procedure described by Chwieroth et al, [8]. In the present work, the imaginary phenomenological potential has a gaussian form, i.e.,

$$
W(r)=W_{0} \exp \left\{-\left(\frac{r-R_{0}}{a}\right)^{2}\right\} .
$$

and, except where otherwise specified, $W_{0}=2 \mathrm{MeV}, R_{0}=1 \mathrm{fm}$ and $a=2 \mathrm{fm}$. These parameters define a $\mathrm{p}+\alpha$ imaginary potential which is reasonably close in magnitude and shape to that found empirically. The aim of the present calculations is only to investigate effects on the resulting absorption due to antisymmetry and no attempt is made to compare RGM calculations with experimental cross-sections. In the following sections, the above imaginary phenomenological potential is included in RGM single channel calculations only, for both $\mathrm{p}+\alpha$ and $\mathrm{d}+{ }^{3} \mathrm{He}, s=1 / 2$ and $s=3 / 2$ cases, and these calculations are denoted "IM".

\subsection{The RGM $S(l)$}

Values of $\left|S_{l}\right|$ and $\arg \left(S_{l}\right)$ for selected calculations are shown in Figs. 11 and 2, for $\mathrm{p}+\alpha$ and $\mathrm{d}+{ }^{3} \mathrm{He}$ respectively. In each case $S(l)$ is displayed for two energies, the energy of the $S(l) \rightarrow V(r)$ inversion and $10 \mathrm{MeV}$ lower in energy to provide an indication of the energy dependence. For $\mathrm{p}+\alpha$, inversions are presented at $40 \mathrm{MeV}$ (in the laboratory frame), well above the reaction threshold at $23 \mathrm{MeV}$ This energy corresponds to a deuteron laboratory energy of $21.67 \mathrm{MeV}$, given the RGM form-factors used here, and the inversions for $\mathrm{d}$ $+{ }^{3} \mathrm{He}$ are mostly presented for this energy.

An additional case, labelled "DIR", is included in Figs. 1 and 2. The DIR potential comprises the sum of the phenomenological imaginary potential and the real RGM direct nuclear potential (obtained by the double folding of the N-N potential with the appropriate wavefunctions, as defined in Ref. [8]). The DIR $S$-matrix is obtained from the direct insertion of the DIR potential into the schrödinger equation, i.e. without antisymmetry. The values of $\arg (S)$ for this DIR case are almost identical to the values obtained by a similar direct calculation without the imaginary potential. The large differences between $S(l)$ for the DIR and the SC cases is well known and has been long understood to arise predominantly due to what is commonly known as the "knock-on exchange" term, [20]. 
Figs. 1 and 12 are discussed in greater detail in the following sections. One obvious feature is the relatively low absorption in the $\mathrm{p}+\alpha$ case, for which $|S(l=2)|>0.85$ at $E=40 \mathrm{MeV}$. This $|S|$ is considerably greater than the empirical values of $\sim 0.65$ and $\sim 0.76$ for $j=3 / 2$ and $j=5 / 2$ respectively, [21, 22]. This deficiency probably arises due to the absence of tensor coupling which establishes the resonance at the reaction threshold and also has a large effect on $|S|$ for the $\mathrm{d}_{3 / 2}$ partial wave immediately above the threshold [22].

\section{3 $S$-matrix to potential inversion}

All the $S$-matrix to potential inversions use the Iterative Perturbative code IMAGO, [23], and the procedure is described in many previous publications (see Ref. [2] and refs therein). The success of the inversion is measured by a phase shift distance, $\sigma^{2}$, between the target (RGM) $S$-matrix, $S^{\text {tar }}$, and the $S$-matrix $S^{\text {inv }}$ corresponding to the potential established by inversion, i.e.

$$
\sigma^{2}=\sum_{\kappa}\left|S_{\kappa}^{\mathrm{tar}}-S_{\kappa}^{\mathrm{inv}}\right|^{2}
$$

and where the index $\kappa$ represents the $l, j$ and energy values of a specified included partial wave. The inversion can then be made for an $S$-matrix at a single energy or for $S$-matrices for a range of energies. Parity dependent potentials are established in the form, $V(r)=V_{1}(r)+(-1)^{l} V_{2}(r)$. Since few partial waves contribute to the inversion for both $\mathrm{p}+\alpha$ and $\mathrm{d}+{ }^{3} \mathrm{He}$, only a small inversion basis of $3-4$ basis functions can be applied for each potential component. Use of a gaussian basis generally leads to the smoothest potentials, but there is necessarily a strong sensitivity to the choice of basis. The resulting uncertainties are most noticeable near $r=0$ where there is an ambiguity between the $V_{1}$ and $V_{2}$ terms. In all cases the smoothest potentials obtained are presented in the following sections.

The inclusion of spin-orbit coupling in the d $+{ }^{3} \mathrm{He}$ channel spin $s=3 / 2$ calculations, presents a new challenge for the iterative perturbative inversion procedure in the form of spin- $3 / 2$ inversion. The inversion method is easily adapted if tensor terms are omitted. The main challenge remaining is then to reproduce phase shifts for more $l, j$ values than the cases with lower spin, but using only central and spin-orbit potentials.

\section{Effects due to a phenomenological imagi- nary potential}

\section{$3.1 \mathrm{p}+\alpha$ scattering}

The comparison of the DIR and IM $S(l)$ for $\mathrm{p}+\alpha$ in Fig 1, shows the contributions of antisymmetry to both $\arg S$ and $|S|$, which, in this case, effectively relate to the real and imaginary local potentials respectively. The introduction of the exchange effects produces a strong parity-dependent effect in $\arg S(l)$, still conspicuous at $40 \mathrm{MeV}$ lab energy, which is little changed by this simple 
introduction of absorption. However, for the s and p waves, $|S|$ obtained for the IM case is significantly closer to unity than $|S|$ for the DIR case. This behaviour is characteristic of the Perey effect, [24], which arises due to knock-on exchange.

Application of IP Inversion to the SC and IM $S(l)$ at $40 \mathrm{MeV}$ yields parity dependent potentials, which are complex for the IM case. These potentials are displayed in Fig, 3, together with the DIR potential. The values of $\arg S$ are practically identical for the SC and IM cases, so that inversion leads to real potentials for these two cases which are graphically indistinguishable. These real potentials are very similar to potentials previously determined from single channel RGM phase shifts for this energy range, [5, 6]. Near the nuclear centre the potential is deeper than the direct potential, reflecting the increase in $\arg S$ for $l<2$ induced by the exchange effects. For $l=2$, $\arg S$ is decreased when antisymmetry is included.

The difference in magnitude, for $r<2 \mathrm{fm}$, between the imaginary potential obtained by inversion and the DIR imaginary component follows in consequence of the behaviour of $|S|$ for $l<2$. If a more surface peaked imaginary potential is used in the RGM, the potential local-equivalent to the resulting phase shifts may become emissive at $r=0$. An imaginary $V_{2}$ term must be included in the inversion to reproduce the phase shifts accurately, but this term is clearly small, that is, much less than the corresponding empirical term. It then appears that the heavy-particle pickup, which causes the real parity-dependence, is of lesser importance to this imaginary component.

The addition of a nucleon-nucleon spin-orbit term into the RGM does not produce any significant changes to the above results. The exchange nonlocality also has very little effect on the spin-orbit terms of the resulting local potential. The real $V_{1}$ spin-orbit term obtained by inversion is almost identical to the RGM direct spin-orbit term, [7]. The inclusion of parity-dependent and imaginary spin-orbit terms in the inversion does decrease $\sigma$, but the resulting potentials are small, i.e. are $<1 \mathrm{MeV}$ for the real $V_{2}$ term and $<0.2 \mathrm{MeV}$ for the imaginary components.

\section{$3.2 \mathrm{~d}+{ }^{3}$ He scattering, $s=1 / 2$}

Figs. I and 2 provide an indication of the differences between the effects of exchange on the $S$-matrices for $\mathrm{d}+{ }^{3} \mathrm{He}$ and for $\mathrm{p}+\alpha$. The local potentials determined by inversion for $\mathrm{d}+{ }^{3} \mathrm{He}$, with channel spin $1 / 2$, at $21.67 \mathrm{MeV}$ are displayed in Fig. 1 for the DIR, SC and IM cases.

Fig. 4 shows a large increase in the depth of the real central $V_{1}$ component for the SC and IM cases compared with the corresponding DIR potential. This decrease presumably arises due to "knockon-exchange", as in the $\mathrm{p}+\alpha$ case. However, the $V_{2}$ component resulting from inversion of the SC and IM cases is entirely positive for $\mathrm{d}+{ }^{3} \mathrm{He}$. The behaviour of $\arg S$ for the $\mathrm{SC}$ case is again almost identical to that the $\mathrm{SC}$ case, except for $l=0$. This small difference in one $\arg S$ value leads to large differences between the SC and IM cases in the real potentials determined by inversion, as shown in Fig. 1 .

The $|S|$ for the IM case significantly differ from the $|S|$ for the DIR case 
for $l<4$, but not in the form of a clear increase in $|S|$ as seen for $\mathrm{p}+\alpha$. This suggests that $|S|$ may be significantly influenced by more than just the knock-on exchange contribution. The resulting $V_{1}$ imaginary term obtained by inversion is very close to the DIR potential and an imaginary $V_{2}$ component is induced which is about half the magnitude of the $V_{1}$ component at $r=1$ $\mathrm{fm}$. If the parity-dependence arises predominantly due to the heavy-particle pick-up then this exchange effect must be considerably greater for $\mathrm{d}+{ }^{3} \mathrm{He}$ than for $\mathrm{p}+\alpha$.

The extended radial structure of the deuteron wavefunction contributes significantly to the differences found between the $\mathrm{p}+\alpha$ and $\mathrm{d}+{ }^{3} \mathrm{He}$ cases. The two systems show a very different behaviour for the divergence of the probability current, $\nabla \cdot \mathbf{j}(\mathbf{r})$ as conventionally defined (and calculated as described in Ref. [25]). When evaluated for the full $s=1 / 2 \mathrm{~d}+{ }^{3} \mathrm{He}$ wavefunction, $\nabla \cdot \mathbf{j}(\mathbf{r})$ is not only substantial for a much wider radial range than the corresponding calculation for $\mathrm{p}+\alpha$, but has also at least twice the magnitude at the maximum value.

A very different pattern of potential features is found if the SC and IM calculations $S(l)$ are based on a simpler deuteron wavefunction consisting of one gaussian alone (with a width $=0.4124 \mathrm{fm}$ ). For this one gaussian case the behaviour of $S(l)$ is qualitatively similar to the values of the full eight gaussian wavefunction, with small differences most noticeable for $l=0$. The real potentials determined for these new SC and IM cases show far more similarities, in shape and magnitude, than the corresponding potentials shown in Fig. ․ Furthermore, the imaginary components obtained by inversion for the new one gaussian IM case also have a different behaviour to that displayed in Fig. 4 . The new imaginary $V_{1}$ component now has a reduction in the magnitude near the nuclear centre compared to the DIR case, similar to the corresponding potential found for $\mathrm{p}+\alpha$. The corresponding $V_{2}$ component has a magnitude of only $0.25 \mathrm{MeV}$, much less than that obtained using the full eight gaussian wave function. If the imaginary parity dependence arises predominantly from heavy-particle pickup, as for the real parity-dependent term, the probability of this exchange is clearly considerably increased by the use of a deuteron wavefunction with a larger radial extent.

The changes in $S(l)$ following the introduction of the phenomenological imaginary term, and consequently in the potentials determined by inversion, are energy dependent. This energy dependence is introduced entirely by the exchange non-locality, since both the nucleon-nucleon potential inserted into the RGM and the phenomenological imaginary term are energy independent. However, a quantitative assessment of the introduced energy dependence is difficult. There is a spurious resonance in the SC case for $l=0$ at about $32 \mathrm{MeV}$, as found by Chwieroth et al, [10], which is effectively removed by including absorption. Energy dependent inversion, using a linear energy dependence for the real components, leads to an accurate reproduction of the IM $\arg S$ for an energy range of $\sim 10 \mathrm{MeV}$, centred at $40 \mathrm{MeV}$. The resulting real components at $40 \mathrm{MeV}$ are very close to the corresponding potentials shown in Fig. \$. However while the imaginary potential input into the RGM has no energy dependence, the resulting absorption shows a strong variation with 
energy for $l \leq 2$, so that $|S|$ cannot be reproduced by inversion using simple linear dependence on energy in the imaginary components. Potentials obtained by applying fixed energy inversion at a series of energies show a decrease with energy in the magnitude of the imaginary $V_{2}$ term in the surface region, while both the imaginary $V_{1}$ and $V_{2}$ terms increase in depth at the nuclear centre as the energy increases.

The inclusion of a simple spin-orbit component in the nucleon-nucleon potential inserted into the RGM calculations has little effect on the results found above. The direct real spin-orbit component obtained from the RGM is much smaller for $\mathrm{d}+{ }^{3} \mathrm{He}$ than for $\mathrm{p}+\alpha$, i.e. $\sim 1 \mathrm{MeV}$ at $r=1 \mathrm{fm}$ compared to $\sim 12 \mathrm{MeV}$ for $\mathrm{p}+\alpha$. Both with and without absorption, the exchange contributions induce a small, but positive, parity dependent component for $\mathrm{d}$ $+{ }^{3} \mathrm{He}$ of $\sim 0.25 \mathrm{MeV}$ at $r=1 \mathrm{fm}$, proportionally much larger than for $\mathrm{p}$ $+\alpha$. Again this suggests a much stronger influence of the exchange terms, particularly the heavy-particle pick-up term, for $\mathrm{d}+{ }^{3} \mathrm{He}$ than for $\mathrm{p}+\alpha$. However, in the IM cases there is no necessity for any significantly non-zero imaginary spin-orbit terms in the local potential.

\section{$3.3 \mathrm{~d}+{ }^{3}$ He scattering, $s=3 / 2$}

The strong dependence of the $\mathrm{d}+{ }^{3} \mathrm{He}$ phase shifts on channel spin reported by Chwieroth et al, 8], is found here in both the phase shifts and local-equivalent potentials, with and without absorption. Chwieroth et al attribute these differences to effects of the Pauli exclusion principle on the two channel spins. As in the $s=1 / 2$ case, significant changes are introduced, in both the RGM $S(l)$ and the resultant local equivalent potentials, when exchange is included in the $s=3 / 2 \mathrm{~d}+{ }^{3} \mathrm{He}$ RGM calculations. The corresponding potentials obtained by inversion for the SC, IM and DIR calculations are displayed in Fig. 5, for a deuteron laboratory energy of $21.67 \mathrm{MeV}$. Fig. 5 also includes the SC potential determined for $\mathrm{d}+{ }^{3} \mathrm{He}$ with channel spin $1 / 2$. The real $V_{1}$ components for the two channel spins in the SC case are qualitatively very similar. However, while the differences for $r<1 \mathrm{fm}$ may be due to ambiguities in the inversion, at larger radii the real $V_{1}$ component is clearly smaller in magnitude for $s=3 / 2$ than for $s=1 / 2$. The real $V_{2}$ component shows a more significant difference between the two channel spins, since these components are opposite in sign for $r>2.5 \mathrm{fm}$. This $V_{2}$ is therefore very different from the $\mathrm{p}+\alpha V_{2}$ component, as predicted by Chwieroth et al.

The values of $\arg (S)$ for the $s=3 / 2$ IM case are almost identical to the values for the $\mathrm{SC}$ case for all $l$ and consequently there is little difference between the SC and IM real potentials. As in previous cases discussed above, the IM $|S|$ again differs considerably from the $|S|$ for the DIR case. The imaginary $V_{1}$ potential consequently decreases in magnitude for $r<3 \mathrm{fm}$, by comparison with the DIR potential, characteristic of a Perey-like effect. Unlike the $\mathrm{p}+\alpha$ case, the corresponding imaginary $V_{2}$ term is relatively large for $r<2 \mathrm{fm}$, and is similar to that obtained for the equivalent potential component for $s=1 / 2$. Therefore, although very different effects are obtained for the two channel spins, in both cases the non-locality generated by the exchange contributions 
must be much stronger for both values of $s$ than in the $\mathrm{p}+\alpha$ case.

The introduction of a spin-orbit term into the single channel RGM produces a much larger inversion problem for $s=3 / 2$. For this $\mathrm{SC}$ case a low inversion $\sigma$ is possible by establishing only central and spin-orbit, $V_{1}$ and $V_{2}$ terms. The central components are then very similar to the equivalent real components shown in Fig. 5. As found in the $s=1 / 2$ case, inversion of the $\mathrm{d}$ $+{ }^{3} \mathrm{He} s=3 / 2 S(l)$ requires a spin-orbit $V_{2}$ term, which is large and negative, i.e. $\sim-0.5 \mathrm{MeV}$ at $r=1 \mathrm{fm}$. This term will also arise due to the strong non-locality resulting from exchange.

\section{Deuteron Distortion Effects on the $\mathrm{d}+{ }^{3} \mathrm{He}$ potential.}

Breakup effects are particularly important in $\mathrm{d}+{ }^{3} \mathrm{He}$ due to the high compressibility of the deuteron. In a detailed study of distortion effects in $\mathrm{d}+$ ${ }^{3}$ He for channel spin $s=3 / 2$, Kanada at al, [26], showed that the specific distortion effects contribute much more strongly to the Pauli favoured states then to the Pauli unfavoured states. These observations were deduced from the phase-shifts, but such behaviour must also be reflected in the potential, especially in the parity-dependence.

In this section the contributions to the $\mathrm{d}+{ }^{3} \mathrm{He}$ potential arising only due to deuteron distortion are considered. The distortion contributions are calculated for both channel spins, although for $s=1 / 2$ the $S(l)$ will not be physically realistic because the $\mathrm{p}+\alpha$ channel coupling is the more energetically favoured configuration. The most accurate results from the RGM have been obtained with up to 15 distortion channels, but a reasonable approximation is found by including only the states corresponding to the lowest 5 energies, [11]. The most significant effect of increasing the number of distortion channels is to reduce the waviness in the energy dependence of $S(l)$, which arises from unphysical dispersion-like resonances. Calculations based on all 5 deuteron states possible with the present choice of deuteron wavefunction (DC) do not have the spurious resonance in the $\mathrm{s}=1 / 2$ channel for $l=0$ at $\sim 19 \mathrm{MeV}$ lab energy. Some smaller and narrower resonance-like features are introduced by the pseudo-state coupling. While the $s=1 / 2$ channel shows a greater energy variation in $|S|$, this variation is mostly of significance outside the energy range of the $S(l) \rightarrow V(r)$ inversion. However, for the $s=3 / 2$ case, a resonance appears for $l=0$ at $\sim 21.5 \mathrm{MeV}$.

Inversion of the DC $\mathrm{d}+{ }^{3} \mathrm{He}$ RGM phase shifts is based on a wider energy range than for the potentials presented in the preceding sections to avoid obtaining unreasonable deductions due to the spurious resonances. Specifically inversion is applied to $S(l)$ for the range of deuteron laboratory energies, $16.6<E_{d}<26.7$. By including a linear energy dependence in the real potential terms, an accurate reproduction of $\arg (S)$ is maintained. However, no simple energy dependence is found for the imaginary components and the resulting potentials provide only an average fit to $|S|$ over the included energy range. 
The potentials, evaluated at an energy of $21.67 \mathrm{MeV}$, for both $s=1 / 2$ and $s=3 / 2$ are presented in Fig. 6, which also shows the potentials determined for the SC cases. The most significant effects of distortion appear at small radii, unsurprisingly as the distortion effects are strongest for low $l$, 226]. The ambiguities between the real $V_{1}$ and $V_{2}$ components makes clear deductions difficult, but for $s=1 / 2$ there is a substantial increase in the $V_{2}$ component which has no parallel feature for $s=3 / 2$. These changes are very similar to the changes induced in the real potential by the introduction of the simple absorption term (the IM case). At large radii the distortion contributions show little effect.

The magnitude of the imaginary terms for $s=3 / 2$ shown in Fig. 6 are ill-determined due to the resonance dominating $|S|$ in the energy range considered. However a strong parity dependent component is required in fixed energy inversion at any energy within this range, and parity-dependent terms are also required for $s=1 / 2$. These imaginary $V_{2}$ components are similar in magnitude but very different in shape for the two channel spins. The two potentials also differ in sign at small radii, notably in the radial region where both real $V_{2}$ components are positive. Clearly here the Pauli effects are strong, but these results also show the contributions of deuteron distortion to be of significance for the potentials for both values of $s$.

\section{Contributions from the reaction channels}

\section{$5.1 \mathrm{p}+\alpha$}

The contributions of the coupling to the $\mathrm{d}+{ }^{3} \mathrm{He}$ channel on the $\mathrm{p}+\alpha \mathrm{RGM}$ potential have previous been investigated at subthreshold energies, [5]. While the coupling contributes just small changes in the potential magnitude, only when the $\mathrm{d}+{ }^{3} \mathrm{He}$ coupling is included is the decrease with energy, found empirically for the real components, obtained for the RGM potentials. At higher energies, the reaction channel coupling contributions to $\arg (S)$ remain small, as seen in Fig. 1, but above $30 \mathrm{MeV}$, the contributions to the absorption become important for the $\mathrm{d}$ and $\mathrm{f}$ waves. For $l<2,|S|$ remains close to unity, similar to the behaviour in the simpler IM case. The introduction of distortion (6C case) in the $\mathrm{d}+{ }^{3} \mathrm{He}$ channel introduces additional uncertainties in the phase shifts due to the necessity to extend the radial integration out to very large radii, [12]. This extra coupling does not, however, lead to significant changes in the $\mathrm{p}+\alpha S(l)$.

The potentials obtained by inversion of both the $\mathrm{CC}$ and the $6 \mathrm{C} S(l)$ are shown in Fig. 7. Also included in this figure is an energy dependent empirical potential, Burzynski (6) of Ref [2] evaluated at $40 \mathrm{MeV}$, which was obtained by inversion of the complex phase shifts of Burzynski et al, [22]. The real, $V_{1}$ and $V_{2}$ components for the $\mathrm{CC}$ and $6 \mathrm{C}$ cases are similar in overall magnitude and shape to the potentials for the $\mathrm{SC}$ case. However the decrease in magnitude of the $V_{2}$ term on introducing the coupling does give an improved agreement with the empirical potential at larger radii. 
Significantly, the channel coupling introduces both a generative imaginary potential at the nuclear centre and a strong parity dependence in the imaginary potential. This parity dependence, much larger than that reported in Sect. 3, probably relates to the influence of the stronger non-locality in the $\mathrm{d}+$ ${ }^{3} \mathrm{He}$ channel on the $\mathrm{p}+\alpha$ channel. The imaginary $V_{2}$ component is of roughly the magnitude found empirically but the shape shows far less agreement with the empirical imaginary $V_{2}$ component than can be seen for all other components in Fig. 17. The magnitude of the generation is far greater than that found empirically, but the results of Sect. 3 suggest that a disagreement in the imaginary components is expected at small radii due to the disagreement of the real components for $r<2 \mathrm{fm}$. The agreement for $r>2 \mathrm{fm}$ maybe slightly fortuitous, since the magnitude of the empirical potential depends strongly on the chosen form of energy dependence, which could not be uniquely established by inversion, [2]. However, the empirical potential shown in Fig. 7 does provide the best fit to the phase shifts of Burzynski et al. A consistent energy dependence cannot be established for the RGM potentials due to the irregularities in the $S(l)$ in both the $\mathrm{CC}$ and $6 \mathrm{C}$ cases. However, the potentials displayed in Fig. 7 for both cases are accurate to within $0.5 \mathrm{MeV}$ over the energy range 35 $-45 \mathrm{MeV}$.

Application of IP inversion to the full RGM calculations, inclusive of spinorbit coupling, leads to central parity dependent potentials which are very close to those shown in Fig. [7. Unlike the calculations of Sect. 3.1 these inversions generate a negative imaginary spin-orbit potential of depth at least $\sim 1 \mathrm{MeV}$ at maximum, in rough agreement with the empirical solution, although the empirical imaginary spin-orbit component is not established very accurately. A reasonable fit to the RGM $S(l)$ is possible without a spin-orbit parity dependence, but, by including the spin-orbit $V_{2}$ terms in the inversion, $\sigma^{2}$ is reduced by a factor of ten. The resulting potentials have a small radial range and are $<1 \mathrm{MeV}$ in magnitude, and again are consistent with empirical results, [2].

\section{$5.2 \mathrm{~d}+{ }^{3} \mathrm{He}$}

The changes in the $\mathrm{d}+{ }^{3} \mathrm{He} S$-matrix arising from introducing the coupling to the $\mathrm{p}+\alpha$ reaction channel, even before the inclusion of deuteron distortion channels, are much larger than the equivalent changes found for $\mathrm{p}+\alpha S(l)$, [9]. This behaviour is illustrated in Fig. 2. The reaction channel coupling contributions to the absorption become more noticeable at the higher of the two energies, predominantly in the $\mathrm{d}$ and $\mathrm{f}$ waves, as in the $\mathrm{p}+\alpha$ case. The further addition of the deuteron pseudo-states produces only small changes in $\arg (S)$, but these states contribute more significantly to $|S|$, particularly for $l=0$ due to the existence of a pseudo-resonance at just below $20 \mathrm{MeV}$.

The potentials obtained by inversion for both the $\mathrm{CC}$ and $6 \mathrm{C}$ cases at the laboratory energy of $21.67 \mathrm{MeV}$ are displayed in Fig. 8 together with the SC potential. The differences noted above for $\arg (S)$ give rise to large differences between the real $\mathrm{CC}$ and $\mathrm{SC}$ potentials. The real $V_{1}$ component for the CC case

is deeper at the centre and has a reduced radial range. For the even $l$-values, a 
large positive contribution to $V_{2}$ at small radii compensates to a certain extent for the change in the real $V_{1}$ term. The apparent oscillation in $V_{2}$ probably arises because the changes in the f-wave introduced with antisymmetry are effectively nullified by the additional effects of channel coupling.

The imaginary components for the $\mathrm{CC}$ case are similar in shape to those of the $\mathrm{p}+\alpha \mathrm{CC}$ imaginary potential, reflecting the strong influence of the channel coupling over the other effects of the exchange non-locality. The $V_{1}$ component is strongly generative at small radii and the $V_{2}$ term has a comparable size. To reproduce the energy dependence of $|S|$ for this CC case, changes in the shape of the imaginary $V_{2}$ term are required. The large difference for $|S(l=0)|$ between the $\mathrm{CC}$ and $6 \mathrm{C}$ cases results in very different imaginary potentials for these two cases. The precise form of these potentials is affected by the pseudo-resonance close by in energy and considerable variations in the imaginary potential are necessary to describe the energy dependence of $|S|$ around this resonance. However the most general feature of these calculations is the decrease in the generation of the $V_{1}$ component near $r=0$ compared to the $\mathrm{CC}$ case.

The full CC calculation, incorporating a spin-orbit interaction, again leads to central local potentials which are very similar to those displayed in Fig. 8. Accurate inversion of these $S(l)$, i.e. to obtain a low $\sigma$, requires parity dependent spin-orbit terms. The resulting imaginary spin-orbit terms are very small, $\sim 0.2 \mathrm{MeV}$ at maximum depth, that is little larger than the equivalent terms reported in Sect. 3.2, so that the additional non-locality produced by the channel coupling has little effect on these terms. However, if the imaginary spin-orbit $V_{1}$ is excluded from the inversion, the resulting imaginary $V_{1}$ central potential becomes oscillatory.

\section{Summary and conclusions}

RGM calculations followed by $S$-matrix to potential inversion have been carried out for $\mathrm{p}+\alpha$ and $\mathrm{d}+{ }^{3}$ He scattering to investigate the effects of antisymmetry and channel coupling on the local potential and particularly the imaginary component. The real part of the $\mathrm{p}+\alpha$ potential has been widely studied in previous investigations, but the $\mathrm{d}+{ }^{3} \mathrm{He}$ potential has received considerably less attention. Here, for probably the first time, $\mathrm{d}+{ }^{3} \mathrm{He}$ potentials are established by accurate inversion of phase shifts, for both channel spins, $s=1 / 2$ and $s=3 / 2$ and a study is presented of the changes in the potentials due to deuteron distortion and channel coupling to the $\mathrm{p}+\alpha$ configuration (for $s=1 / 2)$.

The real parts of the $\mathrm{p}+\alpha$ potential are essentially well determined by the direct contribution plus exchange terms and these terms are only slightly modified by the presence of absorption, whether introduced through channel coupling or via a phenomenological potential. Consequently the strong agreement between the real empirical potential and potentials local equivalent to the single channel RGM established in Ref. [2], up to $65 \mathrm{MeV}$, is unsurprising. However the inclusion of a single reaction channel does improve the agreement 
of the empirical and RGM potentials at the lab. energy of $40 \mathrm{MeV}$ at least, probably to the limit to which the empirical potential is established.

The shape of the imaginary potential is determined by both the exchange non-locality and the particular reaction channels open. Even the simplest form of absorption, for example that represented by introducing a simple imaginary term into the single channel RGM, is significantly distorted by the non-locality arising through exchange alone. For $\mathrm{p}+\alpha$, this effect leads to a reduction in the absorption for low $l$-values and may lead to an emissive potential at the nuclear centre. This behaviour is associated with the Perey effect, which is characteristic of one nucleon exchange. The more complicated exchange nonlocality of antisymmetrisation may also introduce parity dependence into the imaginary components. The effect is not significant for $\mathrm{p}+\alpha$, but does generate a strong imaginary parity dependence for $\mathrm{d}+{ }^{3} \mathrm{He}$. This parity dependence suggests a considerable influence of the heavy-particle pickup contribution in deuteron scattering which appears related to the large radial extent of the deuteron. A fully antisymmetrised calculation inclusive of reaction channels also leads to a strong imaginary parity dependence for both $\mathrm{p}+\alpha$ and $\mathrm{d}+$ ${ }^{3} \mathrm{He}$, although the link to specific exchange effects is more difficult to assign with reaction channel coupling.

The imaginary potential components determined from the reaction channel RGM calculations have roughly the correct magnitude and are qualitatively similar in shape to the empirical imaginary potentials. The imaginary parity dependence is the least accurate component, but the disagreement is not surprising due the underprediction of these RGM calculations for the absorption of certain partial waves. Deuteron distortion contributions have little effects on the $\mathrm{p}+\alpha$ potential and inclusion of additional reaction channels does not correct the underestimation of the d-wave absorption, [12]. However, a critical omission in these RGM calculations may be the lack of a tensor component which would allow coupling to the $s=3 / 2 \mathrm{~d}+{ }^{3} \mathrm{He}$ channel. The inclusion of spin-orbit coupling in the RGM introduces additional components in the local potential, that of most significance being an imaginary spin-orbit term. This component roughly agrees with the imaginary spin-orbit term found in the empirical potential

The description of $\mathrm{d}+{ }^{3} \mathrm{He}$ is more complicated due to the high compressibility of the deuteron. The real potentials for both values of channel spin show the strong increase in magnitude on introduction of exchange effects, as found for $\mathrm{p}+\alpha$ and in many other few nucleon systems. This behaviour is commonly associated with knock-on exchange. The real local potential is also strongly parity dependent, although the precise shape of this parity dependence differs quite significantly for the two channel spins as expected from the phase shift behaviour. The imaginary terms have a similar large dependence on the channel spin, but the resultant shape of the calculated potentials must necessary be qualitative due to the strong dependence on the number of reaction channels included.

The potentials determined in this study differ considerably in shape from the Saxon-woods form used in many standard optical analyses, and expose the limitations of such analyses. For the cases considered here, the imaginary 
central component and both real and imaginary parity dependent forms in particular need to be described by a flexible parameterisation. It is possible that similar properties may be found for other light nucleon systems. The application of inversion to $\mathrm{p}+{ }^{16} \mathrm{O}$ elastic cross-section and analysing power data not only lead to a far better reproduction of the data than could be achieved with conventional optical analyses, but also established imaginary and parity dependent potentials qualitatively similar to those presented here.

This study suggests a need to investigate how far the contributions of a fully antisymmetrised coupled reaction channel analysis can account for the empirical $\mathrm{p}+{ }^{16} \mathrm{O}$ potential, in particular the shape of the imaginary components which show a strong similarity to the potentials found in this work. Also of interest is a empirical analysis of $\mathrm{d}+{ }^{3} \mathrm{He}$, but this presents a much harder proposition. Independent of any tensor components, different parameterisations are necessary for the two channel spins and parity dependence is

expected in both cases. A very comprehensive data set is then necessary to establish all these components to a reasonable accuracy.

\section{Acknowledgements}

The author is very grateful to Dr. R.S. Mackintosh for useful discussions and for a careful reading of this manuscript. Financial support is acknowledged from the EPSRC of the UK, under the grant GR/H00895. 


\section{References}

[1] S.G. Cooper and R.S. Mackintosh, Phys. Rev. C43, (1991) 1001 .

[2] S.G. Cooper and R.S. Mackintosh, Phys. Rev. C54 (1996) 3133.

[3] S.G. Cooper, Nucl. Phys. A618 (1997) 87.

[4] D. R. Thompson and Y.C. Tang, Phys. Rev. C4 (1971) 306.

[5] S.G. Cooper, R.S. Mackintosh, A. Csótó, and R.G. Lovas, Phys. Rev.C 50 (1994) 1308.

[6] S.G. Cooper and R.S. Mackintosh, Nucl. Phys. A592, (1995) 338

[7] I. Reichstein and Y.C. Tang, Nucl. Phys. A158, 529 (1970).

[8] F.S. Chwieroth, Ronald E. Brown, Y.C. Tang and D.R. Thompson, Phys. Rev. C8 (1973) 938.

[9] F.S. Chwieroth, Y.C. Tang and D.R. Thompson, Phys. Rev. C9 (1973) 56.

[10] F.S. Chwieroth, Y.C. Tang and D.R. Thompson, Phys. Lett. 46B (1973) 301.

[11] H. Kanada, T. Kaneko and Y.C. Tang, Nucl. Phys. A504 (1989) 529.

[12] H. Kanada, T. Kaneko and Y.C. Tang, Prog. Theor. Phys. 94 (1995) 1061.

[13] G. Blüge, and K. Langanke, Phys. Rev. C41 (1990) 1191.

- A. Csótó, R. G. Lovas and A. T. Kruppa, Phys. Rev. Lett. 70 (1993) 1389.

[14] G. Blüge, K. Langanke and H.-G. Reusch, "Computational Nuclear Physics 2", Ed. K. Langanke, J.A. Maruhn and S.E. Koonin (1993) (Springer-Verlag, New York).

[15] H. Kanada, T. Kaneko, S. Saito and Y.C. Tang, Nucl. Phys. A444 (1985) 209.

[16] F. Ajzenberg-Selove, Nucl. Phys. A490 (1988) 209.

[17] V.I.Kukulin and G.G. Ryzhikh, Prog. Part. Nucl. Phys. 34 (1995) 397.

- V.I.Kukulin and V.N. Pomerantsev, Sov. J. Nucl. Phys. 50 (1989) 17.

[18] V.I.Kukulin, private communication.

[19] D. R. Thompson, M. LeMere and Y.C. Tang, Nucl. Phys. A286 (1977) 53.

[20] M. LeMere, Y. Fujiwara, Y.C. Tang and Q.K.K. Liu, Phys. Rev. C26, 1847 (1982). 
[21] G.R. Plattner, A.D. Bacher, and H.E. Conzett, Phys. Rev. C5, 1158 (1972).

[22] S. Burzynski, J. Campbell, M. Hammans, R. Henneck, W. Lorenzon, M.A. Pickar, and I. Sick, Phys. Rev. C39, 56 (1989).

[23] S.G. Cooper and R.S. Mackintosh, Users manual for IMAGO, Open University preprint OUPD9201.

[24] R.S. Mackintosh and S.G. Cooper, J. Phys. G:Nucl. Part. Phys.23 (1997) 565 .

[25] S.G. Cooper and R.S. Mackintosh,, Nucl. Phys. A511 (1990) 29

[26] H. Kanada, T. Kaneko, P. N. Shen and Y.C. Tang, Nucl. Phys. A457 (1986) 93. 
Figure 1: For $\mathrm{p}+\alpha$, values of $\left|S_{l}\right|$ and $\arg \left(S_{l}\right)$ calculated at energies of 30 and $40 \mathrm{MeV}$ for the following cases (defined in Sect. 2.1), DIR (full lines), IM (dashed lines), CC (dotted lines) and 6C (dash-dot lines).

Figure 2: For $\mathrm{d}+{ }^{3} \mathrm{He}$, values of $\left|S_{l}\right|$ and $\arg \left(S_{l}\right)$ calculated at energies of 11.67 and $21.67 \mathrm{MeV}$ for the following cases (defined in Sect. 2.1), DIR (full lines), IM (dashed lines), CC (dotted lines) and 6C (dash-dot lines).

Figure 3: For $\mathrm{p}+\alpha$ at a laboratory energy of $40 \mathrm{MeV}$, the real and imaginary, $V_{1}$ and $V_{2}$ components obtained by inversion of the SC $S(l)$ (full lines) and the IM $S(l)$ (dashed lines), compared with the parity independent DIR potential (dotted lines).

Figure 4: For $\mathrm{d}+{ }^{3} \mathrm{He}$ at a deuteron laboratory energy of $21.67 \mathrm{MeV}$ and channel spin $s=1 / 2$, the real and imaginary, $V_{1}$ and $V_{2}$ components obtained by inversion of the SC $S(l)$ (full lines) and the IM $S(l)$ (dashed lines), compared with the parity independent DIR potential (dotted lines).

Figure 5: For $\mathrm{d}+{ }^{3} \mathrm{He}$ at a deuteron laboratory energy of $21.67 \mathrm{MeV}$ and channel spin $s=3 / 2$, the real and imaginary, $V_{1}$ and $V_{2}$ components obtained by inversion of the SC $S(l)$ (full lines) and the IM $S(l)$ (dashed lines), compared with the parity independent DIR potential (dotted lines) and the potential obtained by inversion of the SC $S(l)$ for $s=1 / 2$ (dash-dot lines).

Figure 6: For $\mathrm{d}+{ }^{3} \mathrm{He}$, the real and imaginary, $V_{1}$ and $V_{2}$ components obtained by inversion of (i) the SC $S(l)$ for a deuteron laboratory energy $E_{d}=21.67$ $\mathrm{MeV}$, for both $s=1 / 2$ (full lines) and $s=3 / 2$ (dashed lines), and (ii) the DC $S(l)$ over the energy range $16.6<E_{d}<26.7 \mathrm{MeV}$ for both $s=1 / 2$ (dotted lines) and $s=3 / 2$ (dash-dot lines).

Figure 7: For $\mathrm{p}+\alpha$ at a laboratory energy of $40 \mathrm{MeV}$, the real and imaginary, $V_{1}$ and $V_{2}$ components obtained by inversion of the CC $S(l)$ (dashed lines) and 6C $S(l)$ (dotted lines) compared with the equivalent components determined for the SC case (full lines) and an empirical potential determined in Ref. [2], (Burzynski (6)) labelled "Emp".

Figure 8: For $\mathrm{d}+{ }^{3} \mathrm{He}$ at a laboratory energy of $21.67 \mathrm{MeV}$, the real and imaginary, $V_{1}$ and $V_{2}$ components obtained by inversion of the CC $S(l)$ (dashed lines) and $6 \mathrm{C} S(l)$ (dotted lines) compared with the equivalent components determined for the $\mathrm{SC}$ case (full lines). 
Fig. 1

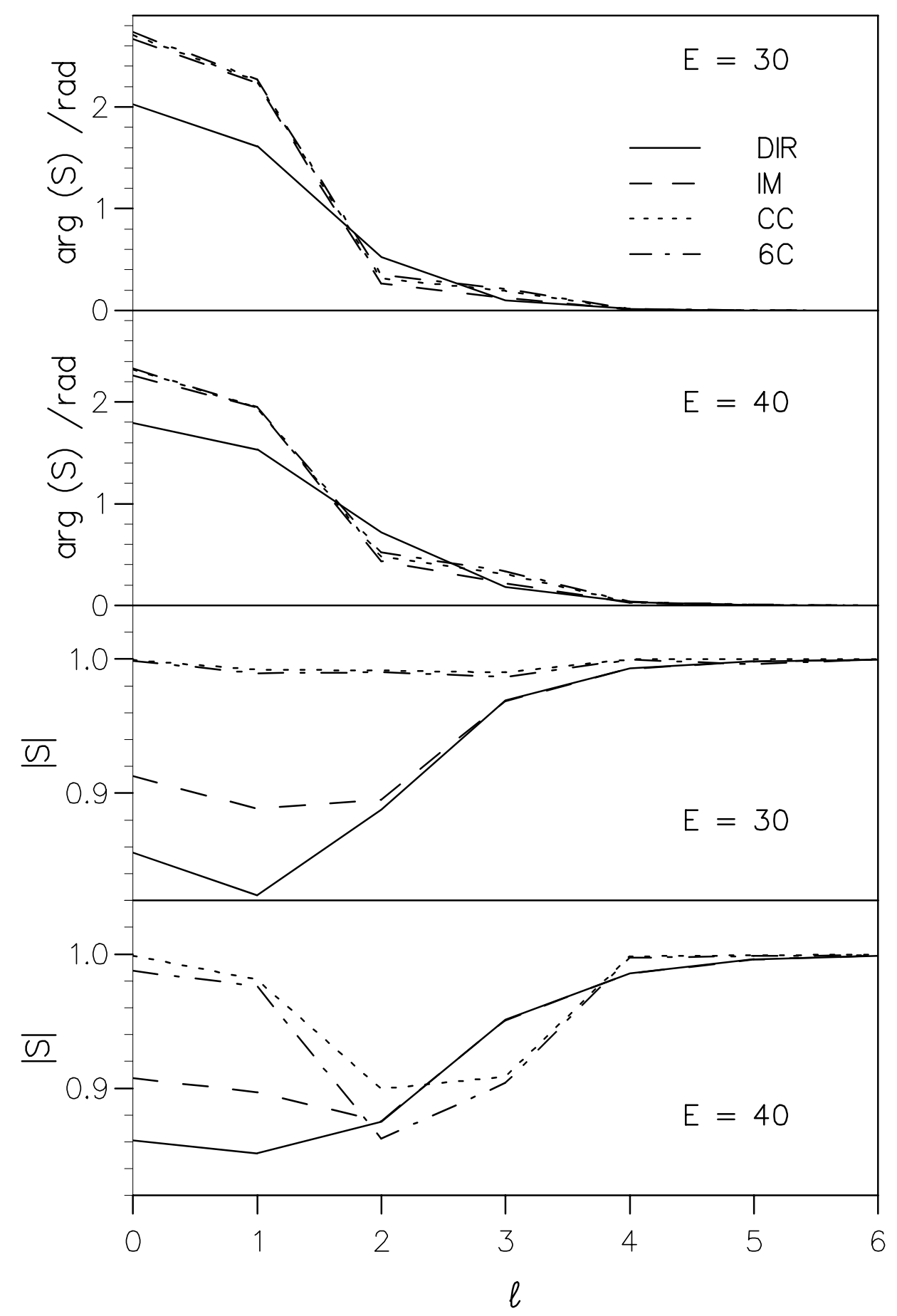


Fig. 2

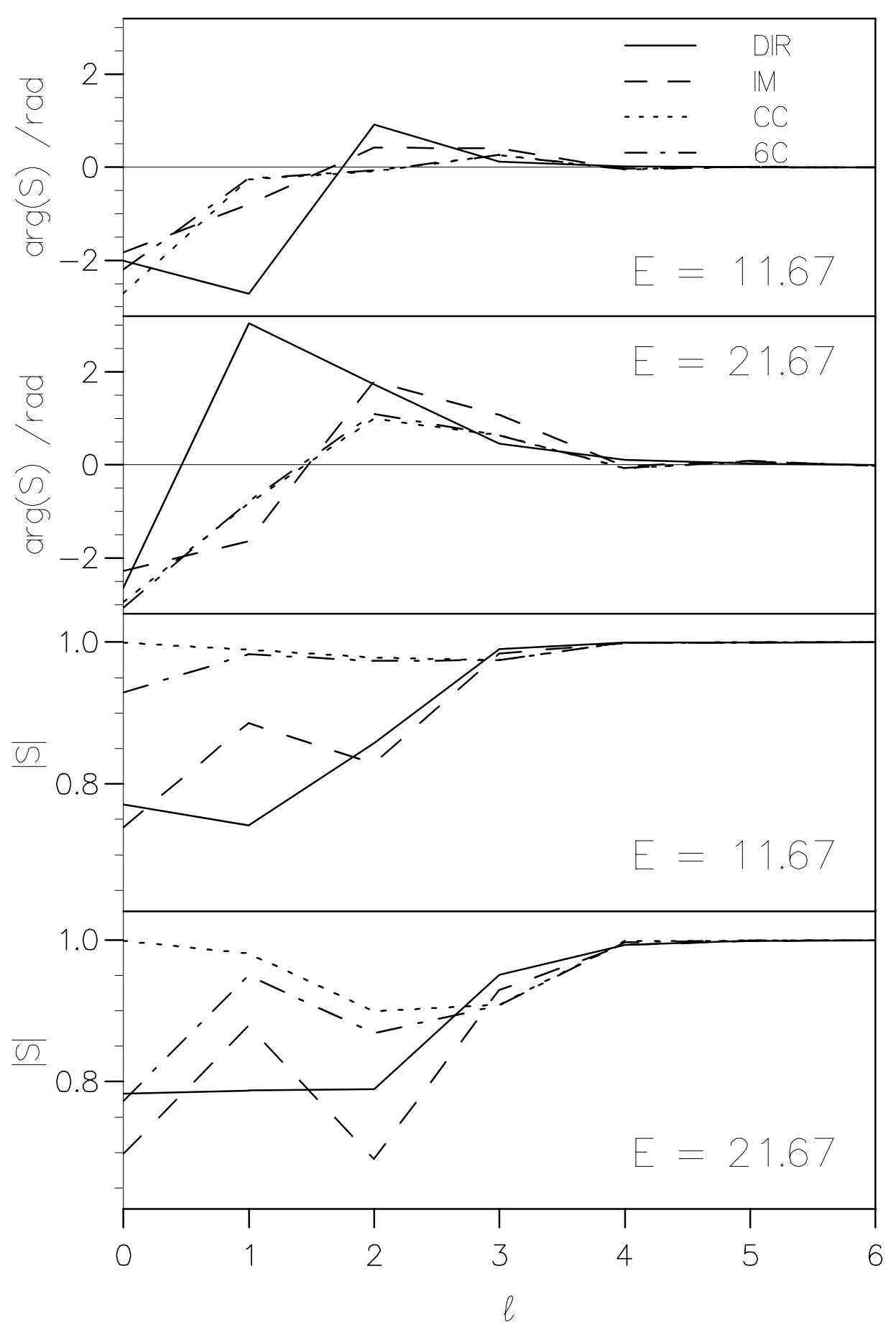


Fig. 3

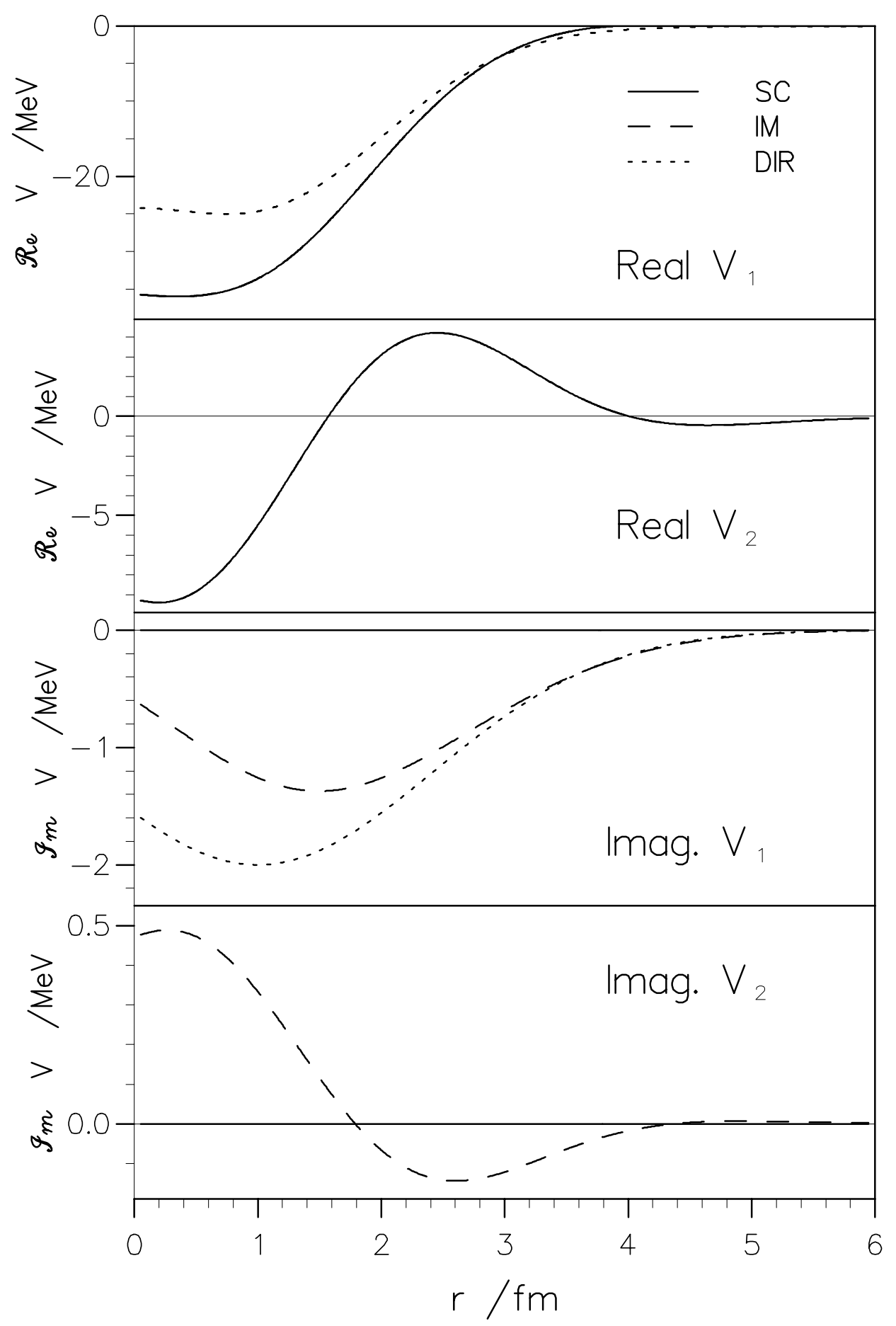


Fig. 4

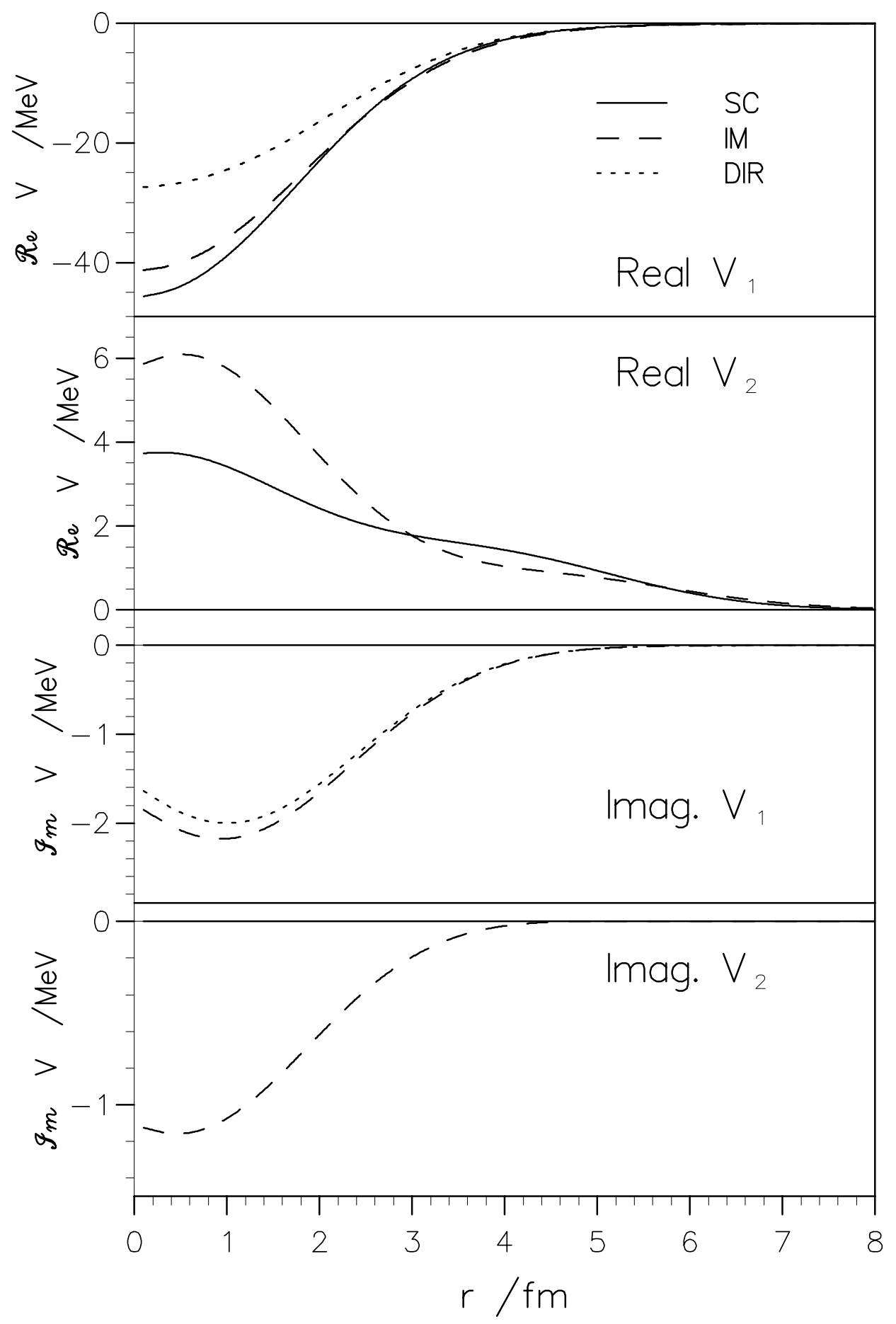


Fig. 5

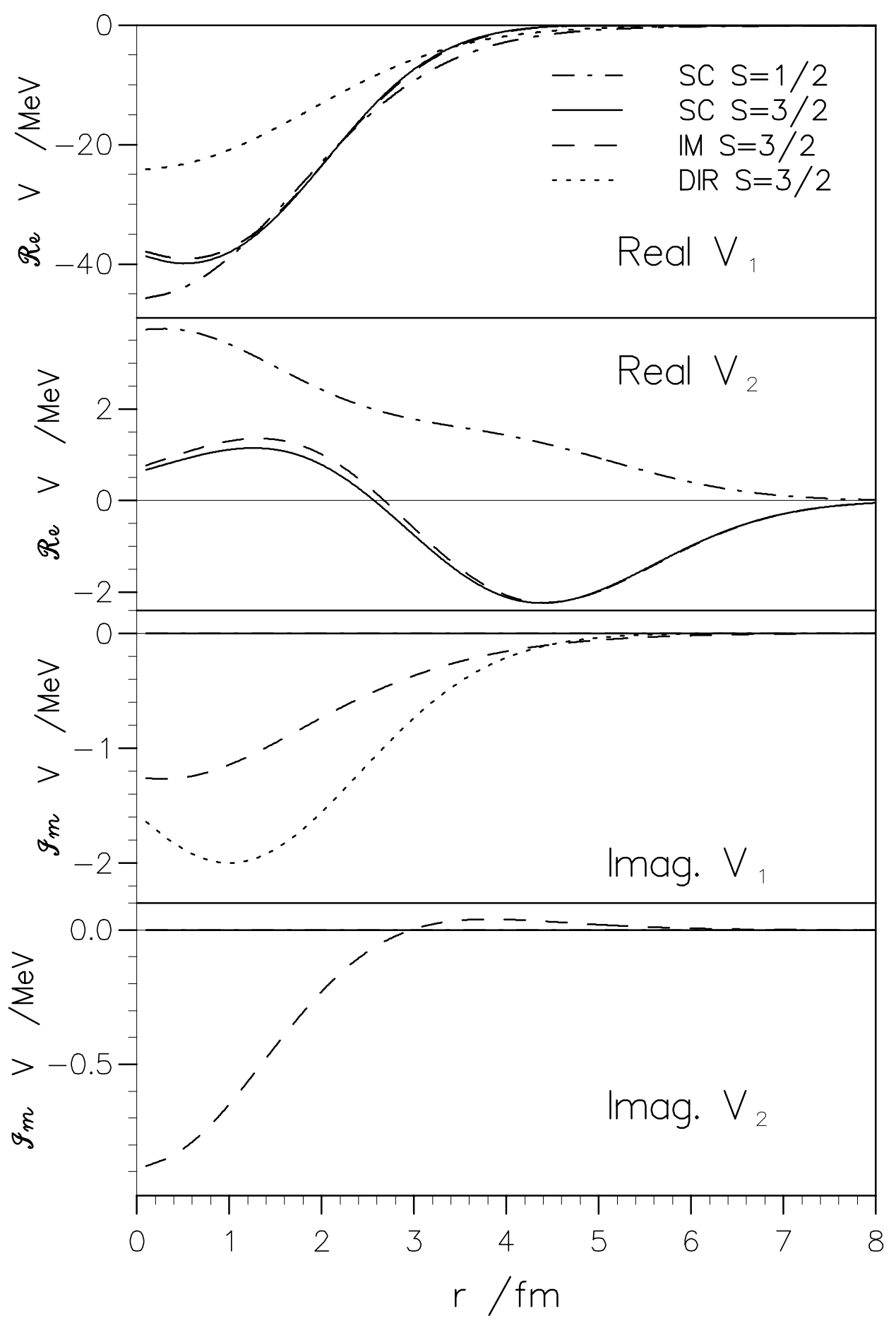


Fig. 6

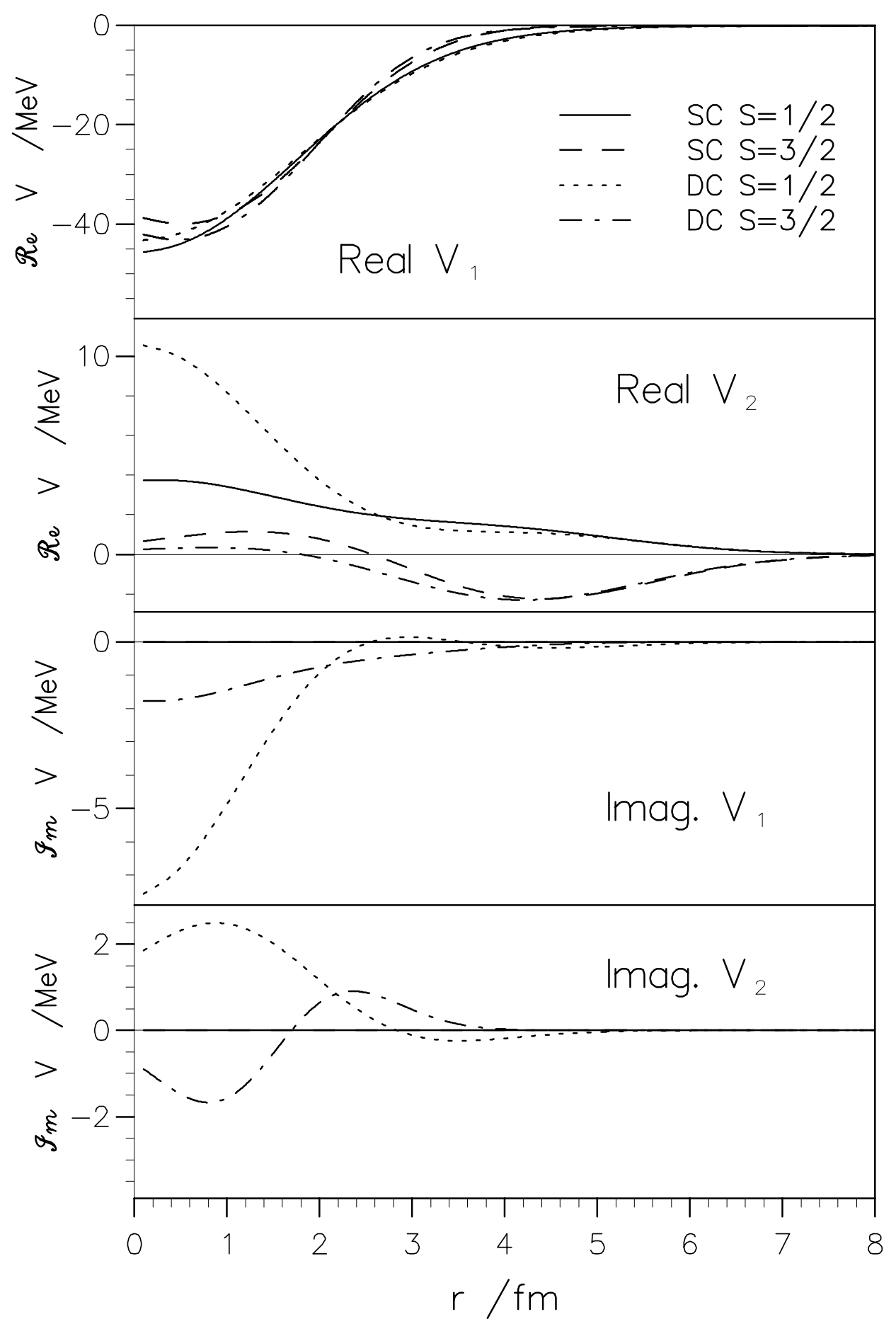


Fig. 7

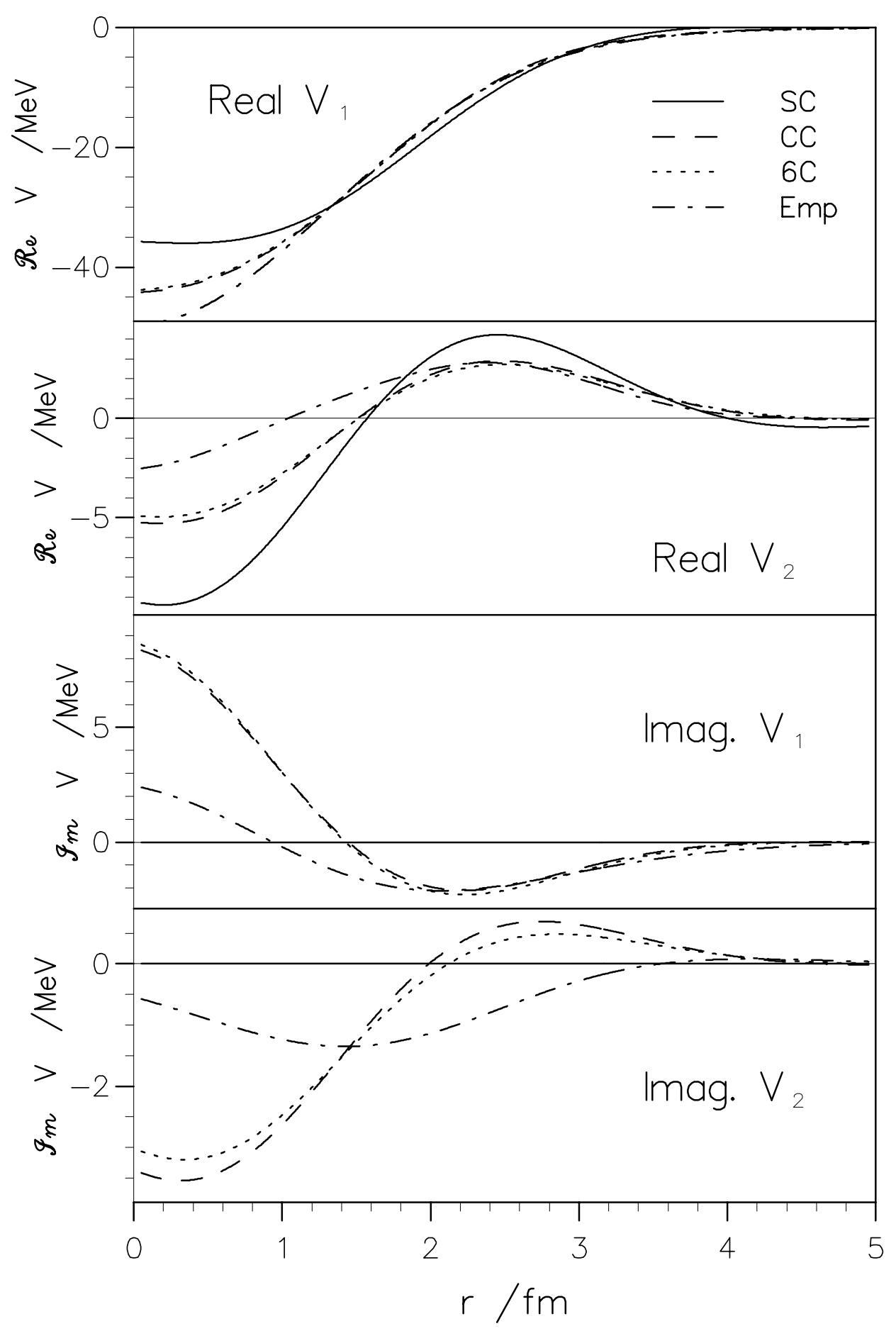


Fig. 8

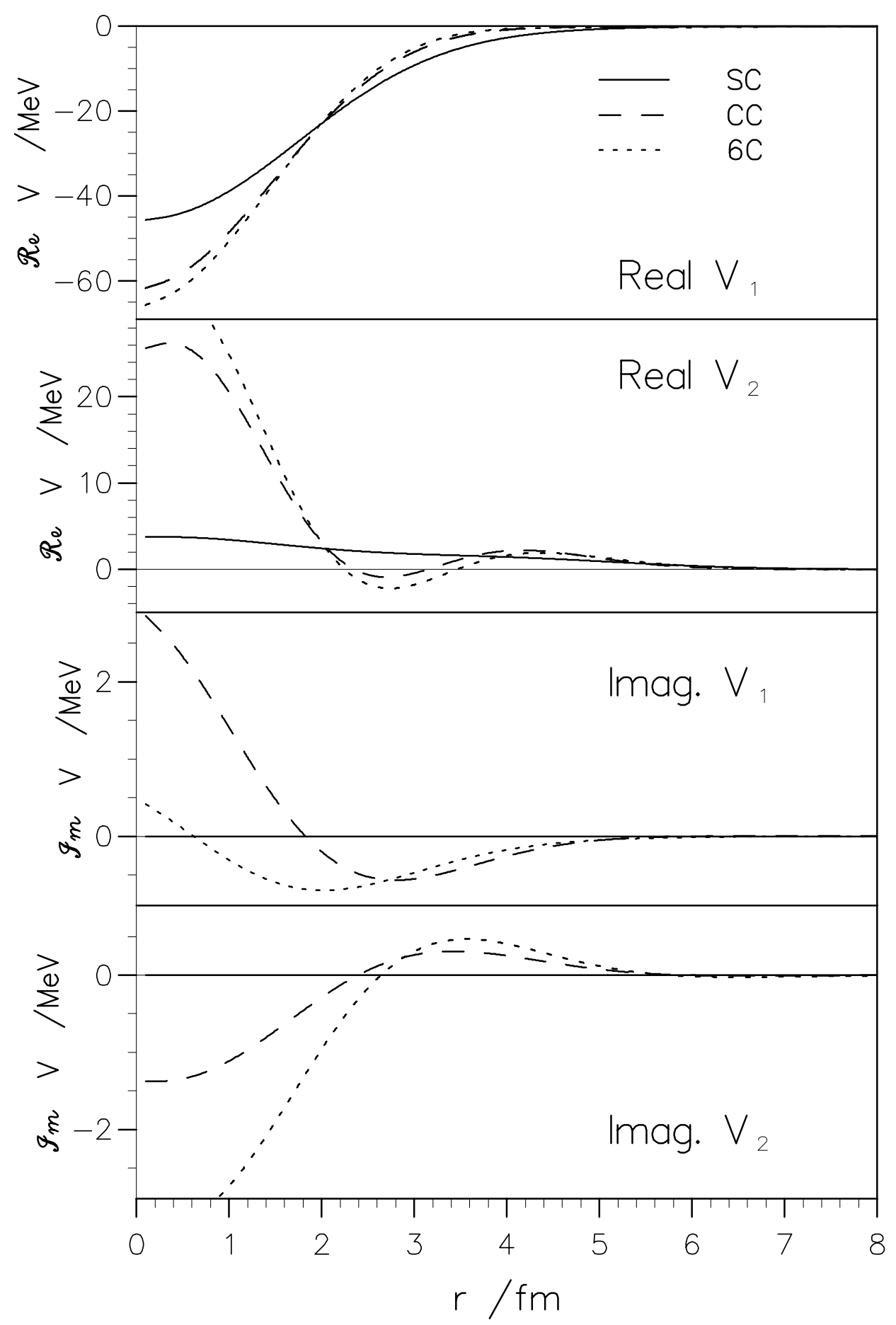

